\title{
Determination of the conditions of spontaneous combustion of a rheologically complex medium inside the continuous infinite cylinder in convective heat transfer case
}

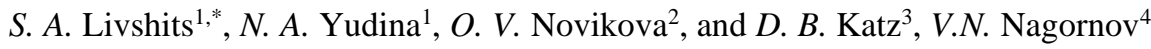 \\ ${ }^{1}$ Kazan State Power Engineering University, Kazan, Russia \\ ${ }^{2}$ Peter the Great St. Petersburg Polytechnic University, Saint-Petersburg, Russia \\ ${ }^{3}$ Kazan Federal University, Kazan, Russia \\ ${ }^{4}$ Belarussian National Technical University, Minsk, Republic of Belarus
}

\begin{abstract}
We obtain the dependence of the relative temperature of a rheologically complex medium depending on the distance to the center of the cylinder. The conditions of spontaneous combustion and the stability of the solutions obtained were investigated, and the approximations of the expressions obtained were carried out. The results obtained in this work allowed us to determine the areas of occurrence of critical flow regimes inside a hollow cylinder under thermal boundary conditions of the 1 st and 3rd kind.
\end{abstract}

\section{Introduction}

In connection with the need to solve various applied problems arising during the design and operation of heat and power plants, the problem of theoretical study of heat and mass transfer processes during the flow of chemically reacting liquids in pipes and channels has come to the fore.

The stationary heat conduction equations in a circular pipe, in channels of complex shapes under various boundary conditions were considered in [1-5]. In [6], the construction of a mathematical model of the polymerization process in a tubular reactor was analyzed in detail.

The purpose of this work is to obtain the dependence of the relative temperature on the distance to the center of the cylinder, to identify the conditions of spontaneous combustion and to determine the stability of the solutions obtained, as well as an approximation of the expressions obtained.

\section{Methods}

In this paper, a mathematical model of D.A. FrankKamenetskii is used

$$
\frac{d^{2} \theta}{d \varepsilon^{2}}+\frac{1}{\varepsilon} \cdot \frac{d \theta}{d \varepsilon}=-F k \cdot \exp [\theta]
$$

This equation is a stationary heat conduction $[7,8]$ equation of an infinite cylinder with a chemical heat source, the reaction rate of which obeys the Arrhenius law, the Frank-Kamenetskii method [9] is used for approximation.

Here:

$$
\begin{gathered}
\varepsilon=\frac{r}{r 1} ; \\
A r=\frac{R \cdot T_{1}}{E} ; \\
\theta[\varepsilon]=\frac{T[\varepsilon]-T_{1}}{A r \cdot T_{1}} ; \\
F k=\frac{Q_{0} \cdot K_{0}}{\lambda \cdot A r} \cdot \exp \left[-\frac{1}{A r}\right] \cdot r 1^{2}
\end{gathered}
$$

$r$ is the distance from cylinder axis; $r l$ is the outer cylinder radius; $A r$ is the Arrhenius number; $R$ is the universal gas constant; $T_{1}$ is the cylinder surface temperature; $E$ is the activation energy of a chemical reaction; $F k$ is the Frank-Kamenetskii criterion; $T[\varepsilon]$ is the absolute temperature; $K_{0}$ is the chemical reaction rate constant; $Q_{0}$ is the thermal effect of a chemical reaction; $\lambda$ is the coefficient of thermal conductivity.

When the cylinder is filled with a condensed phase, the walls are heated [10]. If heat transfer occurs by means of convection, then according to the model of Barzykin V. V. and Merzhanov A. G. the thermal boundary conditions of the 3rd kind are [11]

$$
\left\{\begin{array}{l}
\theta[1]+\frac{\theta_{\varepsilon}^{\prime}[1]}{N u}=0 \\
\theta_{\varepsilon}^{\prime}[0]=0
\end{array}\right.
$$

where $N u$ is the Nusselt criterion.

All calculations are performed using the MATHEMATICA software product [12].

\footnotetext{
* Corresponding author: semen19772004@mail.ru
} 


\section{Results}

The general solution (1) has the form:

$$
\theta[\varepsilon]=\ln \left[\frac{2 c 1^{2} c 2 \varepsilon^{-2+c 1}}{\left(\varepsilon^{c 1}+c 2 F k\right)^{2}}\right]
$$

Analysis of the formula (3) shows that:

$1 . c 1, c 2 \neq 0$.

2. For solid cylinder:

2.1. $0 \leq \varepsilon \leq 1$;

2.2. $c 1=2$, as otherwise $\theta[0]$ is undefined;

2.3. $\theta[\varepsilon]$ monotonously decreasing when $0 \leq \varepsilon \leq 1$; consequently,

$$
\begin{gathered}
\theta[\varepsilon]=\ln \left[\frac{8 c 2}{\left(\varepsilon^{2}+c 2 F k\right)^{2}}\right], \\
\theta_{\max }=\theta[0]=\ln \left[\frac{8}{c 2 F k^{2}}\right],
\end{gathered}
$$

where $c 1, c 2$ are the roots of the system, obtained by substituting (3) into thermal boundary conditions.

As (4) is an even function, it satisfies the last equation from (2). After substituting (4) into the first equation of (2) and some algebraic transformation the system (2) will have the form:

$$
\ln \left[\frac{8 c 2}{(1+c 2 F k)^{2}}\right]-\frac{4}{N u(1+c 2 F k)}=0 .
$$

It seems to be impossible to solve this equation analytically. We denote:

$$
q 2[c 2]=\ln \left[\frac{8 c 2}{(1+c 2 F k)^{2}}\right]-\frac{4}{N u(1+c 2 F k)}
$$

Analysis of (7) showed that:

$1 . c 2>0$;

2. $q 2[c 2]$ has a vertical asymptote when $c 2=0$;

$3 . q 2[c 2]$ has an extremum $(\max )$;

$c 2_{\text {max }}$ is the root of the equation $q 2^{\prime}=0$ :

$$
\begin{gathered}
c 2_{\max }=\frac{2+\sqrt{N u^{2}+4}}{N u F k} \\
q 2_{\max }=\ln \left[N u \frac{\sqrt{N u^{2}+4}-N u}{F k}\right]- \\
-\frac{4}{2+N u+\sqrt{4+N u^{2}}}
\end{gathered}
$$

4. $q 2[c 2]$ monotonously increases when $0<c 2<c 2$ max 5. $q 2[c 2]$ monotonously decreasing when $c 2>c 2$ max , 6. $q 2[c 2]$ has no roots if

$$
q 2_{\max }<0 .
$$

7. $q 2[c 2]$ has the unique root if (spontaneous combustion condition):

$$
q 2_{\max }=0 .
$$

8. $q 2[c 2]$ has two roots if

$$
q 2_{\max }>0 \text {. }
$$

The graphs of $q 2$ [c2] with different $F k$ and constant $N u=3.659$ are shown in figure 1 .

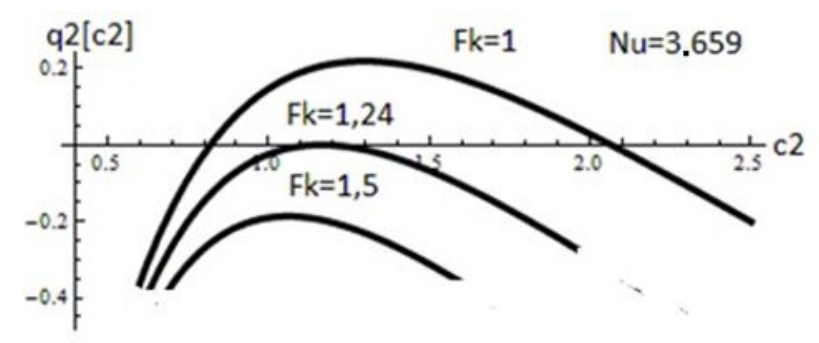

Fig. 1. Graphical interpretation of equation (9) for various $\mathrm{Fk}$ and constant $\mathrm{Nu}=3.659$.

The spontaneous combustion conditions can be transformed by substitution of (9) and (11) and the subsequent solution of the obtained equation

$$
\begin{gathered}
F k=F k_{c r}=\exp \left[\frac{\sqrt{N u^{2}+4}-2-N u}{N u}\right] * \\
* N u\left(\sqrt{N u^{2}+4}-N u\right) .
\end{gathered}
$$

Analysis of (13) shows that:

1. $F k_{c r}$ - monotonously increases;

2. $F k_{c r}-$ has the horizontal asymptote

$$
\operatorname{asimptota}\left[F k_{c r}\right]=2 .
$$

The resulting equation coincides with the condition of spontaneous ignition of an infinite cylinder under thermal boundary conditions of the first kind [9].

To simplify the calculations, we approximate (13):

1. By fractional rational function Pade1/1 [13];

$$
\frac{\text { Pade } 1}{1\left[F_{c r}\right]}=2-\frac{8}{2 N u+3}
$$

2. By fractional rational function Pade2/2;

$$
\frac{\text { Pade } 2}{2\left[F k_{c r}\right]}=2-6 \frac{2 N u+1}{3 N u^{2}+6 N u+4} .
$$

The graphs of (13) - (16) are shown in figure 2

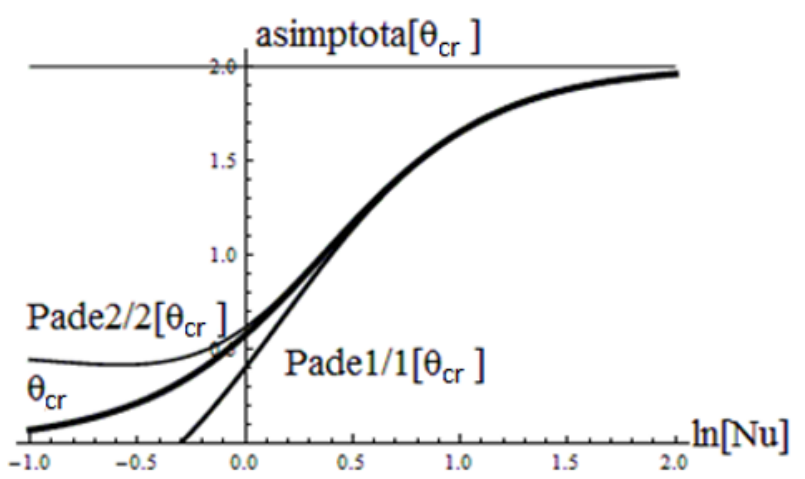

Fig. 2. Graphical Interpretation of Equations (13-16).

For numerical comparison of the approximating curves, we use the absolute value of the relative error 


$$
\begin{gathered}
\delta\left[\text { Pade } 1 / 1\left[F k_{c r}\right]\right]=\left|\frac{\left.F k_{c r}-\text { Pade } 1 / 1\left[F k_{c r}\right]\right]}{F k_{c r}}\right|, \\
\delta\left[\frac{\text { Pade } 2}{2\left[F k_{c r}\right]}\right]=\left|\frac{\left.F k_{c r}-\text { Pade } 2 / 2\left[F k_{c r}\right]\right]}{F k_{c r}}\right|, \\
\delta\left[\text { asimptota }\left[F k_{c r}\right]\right]=\left|\frac{F k_{c r}-a \operatorname{simptota}\left[F k_{c r}\right]}{F k_{c r}}\right| .
\end{gathered}
$$

The graphs of (17) - (19) are show in figure 3.

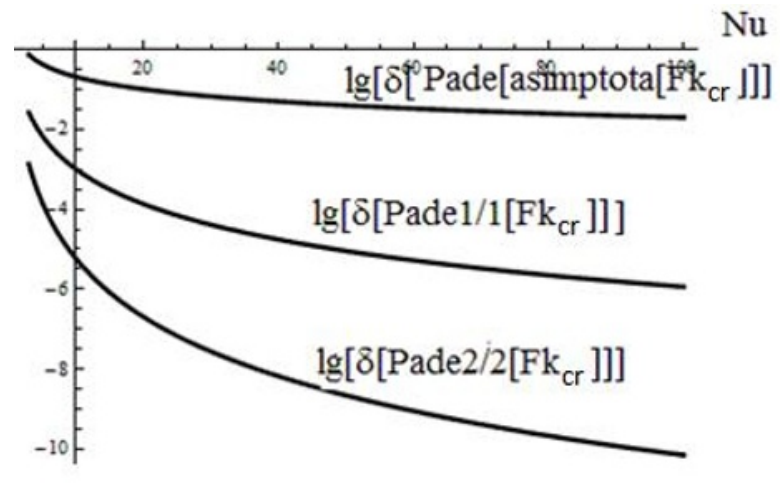

Fig. 3. Graphical Interpretation of Equations (17-19).

By substituting (13) into (8) we can calculate the unique root of (6) with respect to (13):

$$
c 2_{c r}=\frac{\left(2+\sqrt{N u^{2}+4}\right)\left(N u+\sqrt{N u^{2}+4}\right)}{4 N u^{2}} \exp \left[\frac{2+N u-\sqrt{N u^{2}+4}}{N u}\right]
$$

$\mathrm{c} 2$ cr has the horizontal asymptote

$$
\operatorname{asimptota}\left[c 2_{c r}\right]=\frac{1}{2} \text {. }
$$

asimptota $\left[c 2_{c r}\right]$ coincides with $c 2_{c r}$ when thermal boundary conditions of the first kind are held.

With respect to (9) the inequation (10) can be transformed:

$$
F k>F k_{c r}
$$

With respect to (9) the inequation (12) can be transformed:

$$
F k<F k_{c r}
$$

The equation (6) cannot be solved analytically under the conditions (22) and (23). To solve it we will use:

1) the localization of the roots by the analytical method

$$
q 2[c 2]<0 \text { if } \ln \left[\frac{8 c 2}{(1+c 2 F k)^{2}}\right]=0,
$$

which gives us

$$
\begin{gathered}
q 2[c 23]<0 \\
q 2[c 24]<0,
\end{gathered}
$$

where

$$
\begin{aligned}
& \begin{array}{l}
c 3=\frac{F k-4+2 \sqrt{2} \sqrt{2-F k}}{F k^{2}} \\
- \text { is the smaller root of (24), }
\end{array} \\
& c 24=\frac{4-F k+2 \sqrt{2} \sqrt{2-F k}}{F k^{2}} \\
& \text { Consequently, } \quad \text { is the greater root of (24). }
\end{aligned}
$$

$$
\begin{aligned}
& c 23<c 21<c 2_{\max }, \\
& c 2_{\max }<c 22<c 24,
\end{aligned}
$$

where $c 21$ - is the smaller root of (6), c22 is the greater root of (6).

2) The refining of localized roots.

To calculate the $c 21, c 22$ with fixed $F k, N u$ we can use any of numerical methods of solving the non-linear equations [13].

By substituting the roots of (6) into (4) we get the partial solutions of (1) with respect to (2).

- If (22) is valid, then (1) has no partial solutions.

When this condition is held, according to the stationary theory [9] the stationary distribution of temperature is impossible.

- If (13) is held, then (1) has the unique partial solution.

This solution is the critical one

$$
\begin{gathered}
\theta_{c r}[\varepsilon]=\frac{2+N u-\sqrt{4+N u^{2}}}{N u}+ \\
\ln \left[\frac{2\left(2+\sqrt{4+N u^{2}}\right)\left(N u+\sqrt{4+N u^{2}}\right)}{\left(2+\sqrt{4+N u^{2}}+N u \varepsilon^{2}\right)^{2}}\right]
\end{gathered}
$$

The function $\theta_{c r}[\varepsilon]$ has the asymptote

$$
\operatorname{asimptota}\left[\theta_{c r}\right]=2 \ln \left[\frac{2}{1+\varepsilon^{2}}\right] \text {. }
$$

By substituting (27) into (5) and performing the transformations, we get:

$$
\theta_{\text {cr } \max }=\frac{2+N u-\sqrt{4+N u^{2}}}{N u}+\ln \left[\frac{2\left(N u+\sqrt{4+N u^{2}}\right)}{2+\sqrt{4+N u^{2}}}\right] .
$$

The function $\theta_{\text {cr max }}$ has the horizontal asymptote

$$
\text { asimptota }\left[\theta_{\text {cr max }}\right]=\ln [4]
$$

Taking into account the cumbersomeness of (29) we will approximate it with a fractionally rational Pade function to make it simpler [14-16].

$$
\operatorname{Pade} 2 / 2\left[\theta_{\text {cr max }}\right]=\ln [4]-\frac{18}{18 N u^{2}+24 N u+41} \text {. }
$$

The graphs of (29) - (31) are shown in figure 4.

To compare the approximating curves, we use the absolute value of the relative error:

$$
\begin{gathered}
\delta\left[\text { asimptota }\left[\theta_{\text {cr max }}\right]\right]= \\
\left|\frac{\theta_{\text {cr max }}-a \operatorname{asimptota}\left[\theta_{\text {cr max }}\right]}{\theta_{\text {cr } \max }}\right|,
\end{gathered}
$$




$$
\delta\left[\operatorname{Pade} 2 / 2\left[\theta_{c r \max }\right]\right]=\left|\frac{\theta_{c r \max }-\mathrm{Pade} 2 / 2\left[\theta_{c r \max }\right]}{\theta_{c r \max }}\right| .
$$

The graphs of (32) - (33) are shown in figure 5.

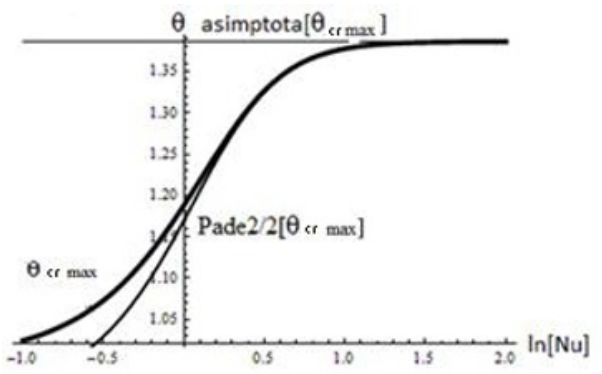

Fig. 4. Graphical Interpretation of Equations (29-31).

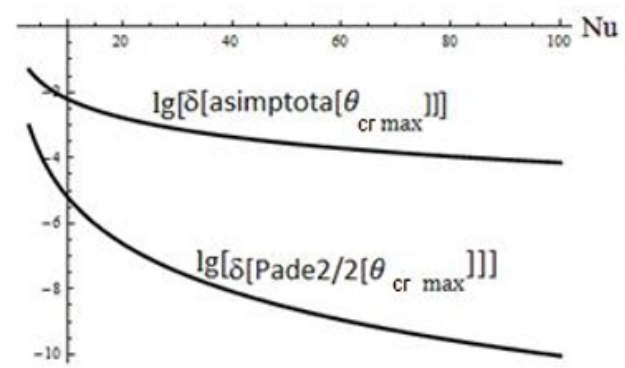

Fig. 5. Graphical Interpretation of Equations (32-33).

If (23) is valid, then (1) has two different partial solutions.

$$
\begin{gathered}
\theta_{1}[\varepsilon]=\ln \left[\frac{8 \cdot c 21}{\left(\varepsilon^{2}+c 21 \cdot F k\right)^{2}}\right], \\
\theta_{2}[\varepsilon]=\ln \left[\frac{8 \cdot c 22}{\left(\varepsilon^{2}+c 22 \cdot F k\right)^{2}}\right],
\end{gathered}
$$

consequently,

$$
\begin{aligned}
& \theta_{1 \text { max }}=\ln \left[\frac{8}{c 21 \cdot F k^{2}}\right], \\
& \theta_{2 \max }=\ln \left[\frac{8}{c 22 \cdot F k^{2}}\right]
\end{aligned}
$$

We should note that:

3.1. $\theta_{1}[\varepsilon]>\theta_{c r}[\varepsilon]>\theta_{2}[\varepsilon]$,

3.2. $\theta_{1}\left[\varepsilon_{\text {max }}\right]>\theta_{c r_{\text {max }}}>\theta_{2}\left[\varepsilon_{\text {max }}\right]$.

6.

The graphs of (27), (35a), (35b) are shown in figure

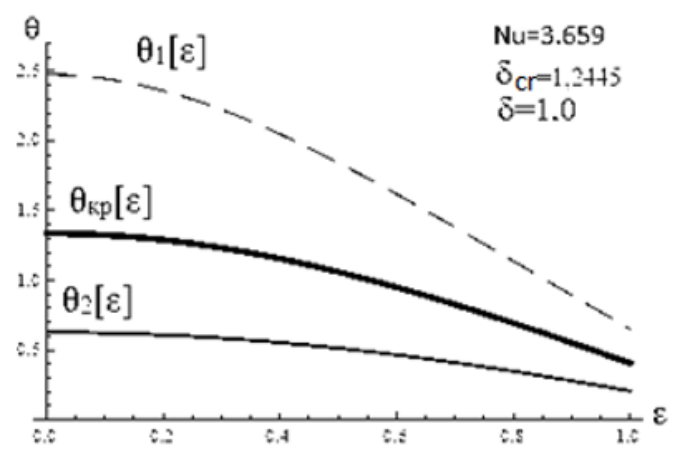

Fig. 6. Graphical Interpretation of Equations (27), (35a), (35b).
$3.3 \theta_{1}[\varepsilon]$ is unstable as $\theta_{1}\left[\varepsilon_{\max }\right]$ when $\delta=0$ has a vertical asymptote and is decreasing monotonously (see figure 7), which is physically impossible.

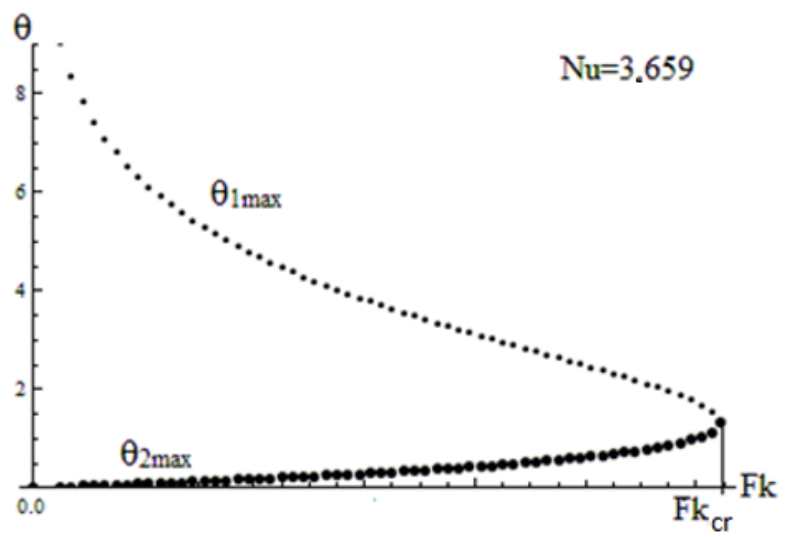

Fig. 7. The dependence of the relative temperature on the value of the Frank-Kamenetskii parameter.

\section{Discussion}

Our research shows that:

1.If $F k<F k_{c r}$ (1) has two partial solutions with respect to the thermal boundary conditions (2). The greater of partial solutions is unstable.

2.If $F k=F k_{c r}$ (1) has the unique partial solution with respect to the thermal boundary condition (2). This solution is critical.

3. If $F k>F k_{c r}$, then according to the stationary theory [9] the stationary distribution of temperature is impossible.

4. Depending on the required accuracy and the value of $N u$, the spontaneous combustion condition can be calculated exactly, or replaced by the spontaneous combustion condition under boundary conditions of the first kind or by the approximating fractional rational Padé function.

5. $\Theta_{\text {cr max }}$ depending on the required accuracy and the value of $N u$, it can be calculated exactly, either replaced by $\lg 4$ or calculated by the approximating rational fractional Pade function.

The use of the approximating fractional rational Pade function significantly reduces the amount of calculations.

\section{References}

[1] R.R. Kantyukov, M.S. Takhaviev, S.A. Livshits, R.V. Lebedev, S.V. Shenkarenko, The Solution of the Stationary Equation of Heat Conductivity with the Chemical and Dissipative Heat Source in an Infinite Circular Pipe for a Newtonian Fluid // Vestnik Kazanskogo tekhnologicheskogo universiteta. The Herald of Kazan Technological University. 18(11), 200-205 (2015)

[2] R.R. Kantyukov, M.S. Takhaviev, S.A. Livshits, R.V. Lebedev, S.V. Shenkarenko, The Solution of the Stationary Equation of Heat Conductivity with the Chemical Source with the Boundary Thermal Conditions 
of the Third Kind in an Infinite Circular Pipe // Vestnik Kazanskogo tekhnologicheskogo universiteta. The Herald of Kazan Technological University. 18(9), 222-225 (2015)

[3] R.R. Kantyukov, M.S. Takhaviev, S.A. Livshits, R.V. Lebedev, S.V. Shenkarenko, The Analytical Research for the Presence of Bifurcation Effects in the Flow of Nonlinear Viscous Fluids in Channels of Complex Geometry // Vestnik Kazanskogo tekhnologicheskogo universiteta. The Herald of Kazan Technological University 18(4), 223-225 (2015)

[4] D.M. Clemmov, An extension of the theory of thermal explosion and its application to the oscillatory burning of explosives // Transactions of the Faraday Society 52, 385-396 (1956)

[5] A.G. Merzhanov, Combustion and explosion processes in physical chemistry and technology of inorganic materials. Russ. Chem. 72(4), 323-345 (2003)

[6] Yu.S. Sidorova, Ye.K. Vachagina, V.V. Plotnikov, R.R. Kantyukov, M.S. Takhaviev, On constructing a mathematical model of the process of heterophase polymerization of ethylene in a tubular reactor // Vestnik Kazanskogo tekhnologicheskogo universiteta. The Herald of Kazan Technological University. 18(4), 231-237 (2015)

[7] R.C. Reid, The properties of gases and liquids, B E Poling. - USA, New York: McGraw-Hill, 753 (1987)

[8] Y.S. Touloukian, Thermophysical properties of matter, New York: Plenum Press, 197

[9] D.A. Frank-Kamenetskii, Diffusion and Heat Exchange in Chemical Kinetics - Princeton, Princeton University Press 503 (1955)

[10] H.Z. Baracat, I.A. Clark, Analytical and experimental study of the transient laminar natural convection flows in partially filled containers. // Proc. Ill Int. Heat Transfer Conf. Chicago, 2 (1966)

[11] V.V. Barzykin, A.G. Merzhanov, The Boundary problem in the thermal explosion theory. -Dokl. AN SSSR, 120(6), 1271 (In Russian) (1958)

[12] V.P. Dyakonov, Mathematica training course SPB: Piter, - 656 (2001)

[13] P.V. Melentev, Priblizhennye vychisleniya Moscow, FML 388 (1962)

[14] A.J. Chorin, A numerical method for solving incompressible viscous flow problems. // Comput. Phys. 2 (1967)

[15] Yu.A. Labych, A.P. Starovoitov, "Trigonometric Padé approximants for functions with regularly decreasing Fourier coefficients", Sb. Math., 200(7), 1051-1074 (2009)

[16] G.A.Jr. Baker, P. Graves-Morris, Pade Approximants, Cambridge University Press 760 (1966) 